\title{
Research on Real-Time Simulation System of Ship Motion Based on Simulink
}

\author{
Yingfei Zan ${ }^{1}$, Duanfeng $\mathrm{Han}^{*}, 1$, Lihao Yuan ${ }^{1}$, Minghao Liu ${ }^{1}$ and Zhaohui $\mathrm{Wu}^{2}$ \\ ${ }^{1}$ College of Shipbuilding Engineering, Harbin Engineering University, Harbin, 150001, China \\ ${ }^{2}$ Offshore Oil Engineering Co., Ltd., Tianjin, 300451, China
}

\begin{abstract}
This paper puts forward a development method of ship motion simulation system that can conduct UDP communication in real time. This system is based on Simulink in order to meet the need of modular and fast ship motion simulation systems. First, the mathematics models of ship motion are set up based on the modular ideology, which includes thruster model and models of wind, wave and current. Then, real time simulation module using S-fun function is developed, and built into executable program. This could realize real time communication between ship motion simulation system and visual simulation system and induce real time communication between ship motion system and ship simulation control system. In this way, it can save time and cost when developing the simulation system. This method has been used successfully in the development of real time simulation system of some engineering ship.
\end{abstract}

Keywords: Real time simulation, Ship motion, Simulink, UDP communication.

\section{INTRODUCTION}

Real-time ship motion simulation system based on ship motion kinematic mathematical model utilizes computer simulation and human-computer interaction technology to acquire real-time interactive simulation of process of sailing and operation. The system possesses the ability of testing performance of ship motion, training ship operators and obtaining prediction and assessment of on-sea operation schemes, shortening the periods and costs of real-ship training and reducing the possibility of risky on-sea operation and damaged marine equipment, while effectively securing the safety of workers and equipment. However, adding UDP protocol module and real-time control mode into $\mathrm{C}$ mode transferred from Simulink model can attain executable program, greatly increasing the amount of work in postprocessing and program changing [1]. In this paper, modular ship motion mathematical model is established including propeller model and wind, wave and current environmental models. UDP-based protocol is utilized to communicate directly with other systems and S-fun to develop real-time simulation module [2-4]. Finally, Simulink directly compiles an executable file to achieve a smooth transition from Simulink to real-time communication with other systems without post-processing and a perfect link between design phase and implementation phase is obtained.

\section{SHIP MOTION MODEL}

\subsection{Establishment and Transformation of Coordinate}

In order to describe ship motion at sea, two right-hand cartesian coordinates are needed: North-East-Down

*Address correspondence to this author at the College of Ship Building Engineering, Harbin Engineering University, Harbin, 150001, China; Tel: +86-451-82519910; Fax: +86-451-82518443;

E-mail: handuanfeng@hrbeu.edu.cn coordinate frame $O_{0}-x_{0} y_{0} z_{0}$, which is an inertial frame for describing movement of ship; the other one is body-fixed kinematic frame $G-x y z$, which is fixed to the ship with its origin at the center of gravity. These two frames are elaborated below[5]:

\subsubsection{North-East-Down (NED) Coordinate System}

North-East-Down (NED) coordinate system $O_{0}-x_{0} y_{0} z_{0}$ is fixed to the surface of earth. When $t=0$, origin of fixed coordinate is on the surface of ocean, $O_{0} x_{0}$ axis points towards true north, $O_{0} y_{0}$ axis points towards true east, $O_{0} z_{0}$ axis points down, as in the case of direction of gravity. Fig. (1) illustrates reference frame relationship, $\psi_{T}, \psi_{W}, \psi_{C}$ as wind angle, wave angle and current angle, respectively.

\subsubsection{Body-Fixed Reference System}

Body-fixed reference system $G-x y z$ moves with the ships' body. Origin of the coordinate is at the center of gravity $G$; $G x$ axis is along the longitudinal (stern to bow), and according to the right-hand rule, $G y$ axis is along transversal (port to starboard), and $G z$ axis points down coinciding with gravity. $\beta$ is the angle between the direction of ship's movement and $G x$ axis, called drift angle. Value of drift angle is positive when ship's speed $\vec{V}$ rotates clockwise to $G x$ axis.

Ship motion model is divided into a main module (6 DOF ship motion mathematical module) and five component modules (hydrodynamic module, wave force module, wind force module, current force module and thruster module) as shown in Fig. (2). 
Table 1. Definition of Ship Motion and Force.

\begin{tabular}{|c|c|c|c|c|}
\hline DOF & Motion/Rotation Direction & Forces/Moments & Linear/Angular Velocities & Positions/Euler Angles \\
\hline \hline 1 & surge & $X$ & $u$ & $x$ \\
\hline 2 & sway & $Y$ & $w$ & $y$ \\
\hline 3 & heave & $Z$ & $p$ & $z$ \\
\hline 4 & roll & $K$ & $q$ & $\theta$ \\
\hline 5 & pitch & $M$ & $\Psi$ \\
\hline 6 & yaw & $N$ & & \\
\hline
\end{tabular}

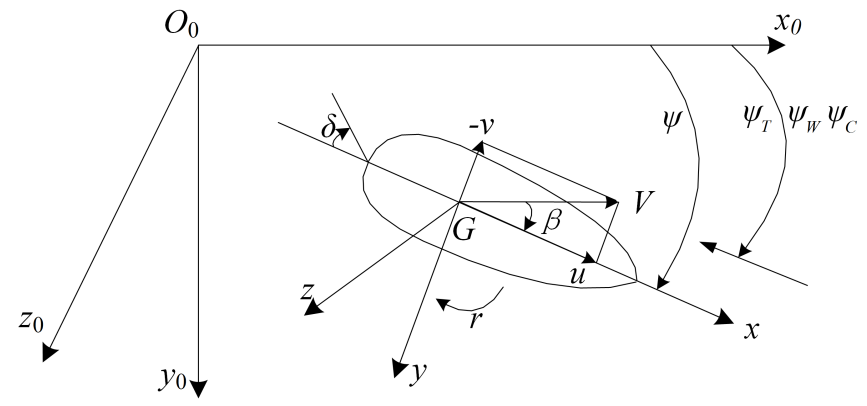

Fig. (1). Reference frame relationship.

\subsection{Six DOF Ship Motion Mathematical Module}

In maneuvering, a ship's motion is in 6 degrees of freedom (DOF). Ship motion and force are defined in Table $\mathbf{1 .}$
According to Newton's theorem of mass center movement and moment of momentum, equation of ship motion is [6]:

$$
\left\{\begin{array}{l}
\dot{\eta}=J(\eta) v \\
M_{R B} \dot{v}+C_{R B}(v) v+M_{A} \dot{v}_{r}+C_{A}\left(v_{r}\right) v_{r}+D\left(v_{r}\right) v_{r}+G \eta=\tau_{R B}
\end{array}\right.
$$

where:

$$
J(\eta)=\left[\begin{array}{ccclcc}
c \psi c \theta & -s \psi c \phi+c \psi s \theta s \phi & s \psi c \phi+c \psi c \phi s \theta & 0 & 0 & 0 \\
s \psi c \theta & c \psi c \phi+s \phi s \theta s \psi & -c \psi s \phi+s \theta s \psi c \phi & 0 & 0 & 0 \\
-s \theta & c \theta s \phi & c \theta c \phi & 0 & 0 & 0 \\
0 & 0 & 0 & 1 & s \phi t \theta & c \phi t \theta \\
0 & 0 & 0 & 0 & c \phi & -s \phi \\
0 & 0 & 0 & 0 & s \phi / c \theta & c \phi / c \theta
\end{array}\right]
$$

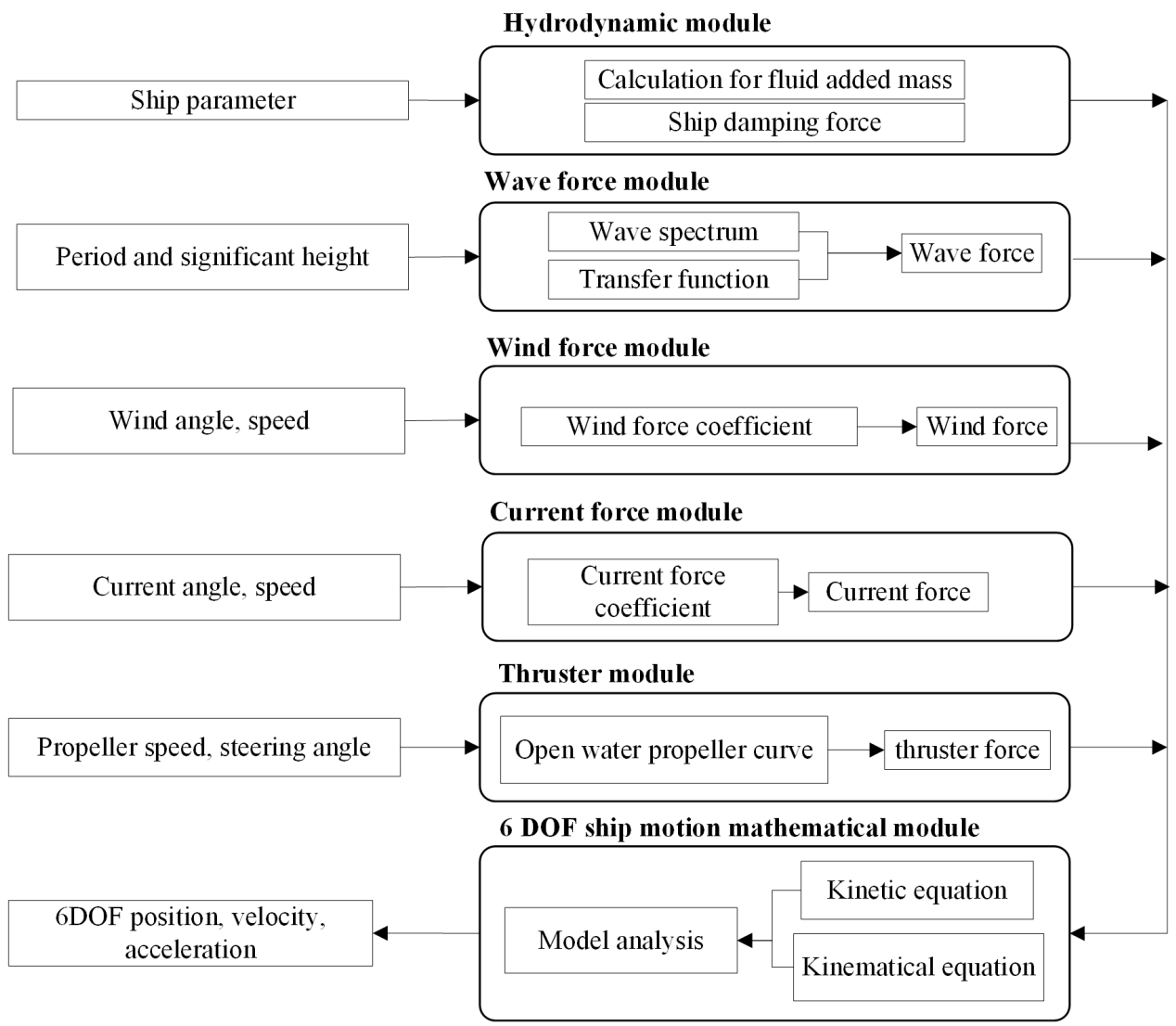

Fig. (2). Framework of motion model. 
$\eta=[x, y, z, \phi, \theta, \psi]^{T}, v=[u, v, \omega, p, q, r]^{T} ;$

$M_{R B}$ is the rigid-body inertia matrix;

$C_{R B}$ is the Coriolis-centripetal matrix;

$M_{A}$ is the added mass inertia matrix;

$C_{A}\left(v_{r}\right)$ is the added mass Coriolis-centripetal matrix;

$D\left(v_{r}\right)$ is the damping matrix

$G$ is the stiffness matrix;

$v_{r}=v-v_{c}$ is the relative velocity;

$\tau_{R B}=[X, Y, Z, K, M, N]^{\mathrm{T}}$ is the environmental disturbance from wind, waves and currents.

where: $c(\bullet)=\cos (\bullet), \mathrm{s}(\bullet)=\sin (\bullet)$

\subsection{Hydrodynamic Module}

Added mass and damping coefficient are obtained by model tests, while lack of data of ship model empirical method is also practical.

\subsubsection{Plane Added Mass Calculation}

Zhou Zhao-ming applied multivariate regression analysis to Yuan Liang-cheng three spectrum, offering a method for estimating added mass of plane motion [7].

$$
\begin{aligned}
\frac{\lambda_{11}}{m}= & \frac{1}{100}\left[0.398+11.97 C_{b}\left(1+3.73 \frac{d}{B}\right)-2.89 C_{b} \frac{L}{B}\right. \\
& \left.*\left(1+1.13 \frac{d}{B}\right)+0.175 C_{b}\left(\frac{L}{B}\right)^{2}\left(1+0.541 \frac{d}{B}\right)-1.107 \frac{L}{B} \frac{d}{B}\right] \\
\frac{\lambda_{22}}{m} & =0.882-0.54 C_{b}\left(1-1.6 \frac{d}{B}\right)-0.156 \frac{L}{B}\left(1-0.673 C_{b}\right) \\
& +0.826 \frac{d}{B} \frac{L}{B}\left(1-0.678 \frac{d}{B}\right)-0.638 \frac{d}{B} \frac{L}{B}\left(1-0.669 \frac{d}{B}\right) \\
\sqrt{\frac{\lambda_{66}}{m L^{2}}} & =\frac{1}{100}\left[33-76.85 C_{b}\left(1-0.784 C_{b}\right)\right. \\
& \left.\left.+3.43 \frac{L}{B}\left(1-0.63 C_{b}\right)\right]-1.107 \frac{L}{B} \frac{d}{B}\right]
\end{aligned}
$$

where:

$m$ is the ship mass

$C_{b}$ is the block coefficient

$d$ is the ship draught

$L$ is the ship length

$B$ is the ship breadth

\subsubsection{Rolling Added İnertial Moment Calculation}

Rolling added inertial moment can be calculated as [8]:

$I_{x}+\lambda_{44}=\frac{\Delta}{g} \rho_{\varphi}^{2}$

where:
$\Delta$ is displacement;

$g$ is acceleration of gravity;

$\rho_{\varphi}$ is inertial radius for rolling with consideration of added mass, as a result of product of breadth $B$ and empirical coefficient $c$. For passenger ships, $c=0.40 \sim 0.435$. For cargo ship, when full load $c=0.32 \sim 0.35$, then in ballast $c$ $=0.37 \sim 0.40$. According to the requirement of Japanese stability norm, for full form ship, Formula (4) is the method for calculation:

$c=0.3725+0.0227 \frac{B}{d}-0.0043 \frac{L}{100}$

For fine form ship, it is:

$c=0.3085+0.0227 \frac{B}{d}-0.0043 \frac{L}{100}$

\subsubsection{Heave Added Mass and Pitch Added Inertial Moment Calculation}

Heave added mass and pitch added inertial moment can be estimated by F. Tasai's empirical method:

$\left\{\begin{array}{l}\lambda_{33}=0.8 \frac{B}{2 d} C_{w} m \\ \lambda_{55}=0.83 \frac{B}{2 d} C_{p}{ }^{2}(0.25 L)^{2} m\end{array}\right.$

where:

$C_{w}$ is the waterplane coefficient;

$C_{p}$ is the prismatic coefficient.

\subsubsection{Plane-Motion Viscous Hydrodynamic Coefficient}

Estimation for plane-motion viscous hydrodynamic force is obtained by [9]:

$$
\left\{\begin{array}{l}
X_{N}=X(u)+X_{v v} v^{2}+X_{v r} v r+X_{r r} r^{2} \\
Y_{N}=Y_{v} v+Y_{r} r+Y_{v v \mid} v|v|+Y_{v|r|} v|r|+Y_{r|r|} r|r|+Y_{v v r} v^{2} r+Y_{v r r} v r^{2} \\
N_{N}=N_{v} v+N_{r} r+N_{v|v|}|v| v+N_{r|r|} r|r|+N_{v v r} v^{2} r+N_{v r r} v r^{2}
\end{array}\right.
$$

$X(u)$ is resistance when the ship is in direct route; $X_{v v}, X_{v r}$, $X_{r r}$ are derivatives of longitudinal nonlinear hydrodynamic force; $Y_{v}, Y_{r}, Y_{v|v|}, Y_{v|r|}, Y_{r|r|}, Y_{v v r}, Y_{v r r}$ are derivatives of transverse linear and nonlinear hydrodynamic force; and $N_{v}$, $N_{r}, N_{v|v|}, N_{r|r|}, N_{v v r}, N_{v r r}$ are derivatives of heading linear and nonlinear hydrodynamic force.

\subsubsection{Estimation for Rolling Damping Moment}

Roll damping moment follows linear relation with roll angular velocity, $L_{\dot{\varphi}}$ is rolling damping coefficient while rolling angle is small. The method for estimation is :

$L_{\dot{\varphi}}=\mu_{\varphi} \sqrt{\left(I_{x}+\lambda_{44}\right) \cdot \Delta \cdot G M}$ 
where:

$G M$ is the metacentric height;

$\mu_{\phi}$ is the non-dimensional attenuation coefficient.

\subsubsection{Heaving and Pitching Viscous Hydrodynamic Calculation}

Heave viscous hydrodynamic coefficient $Z_{V}$ is:

$Z_{V}=-Z_{\dot{z}} \dot{z}-Z_{z} z-Z_{\ddot{\theta}} \ddot{\theta}-Z_{\dot{\theta}} \dot{\theta}-Z_{\theta} \theta$

Pitch viscous hydrodynamic moment $M_{V}$ is:

$M_{V}=-M_{\dot{\theta}} \dot{\theta}-M_{\theta} \theta-M_{\ddot{z}} \ddot{z}-M_{\dot{z}} \dot{z}-M_{z} z$

Combining F. Tasai's empirical method (9) with hydrodynamic derivatives in (10) [10].

Heave hydrodynamic derivative:

$$
\left\{\begin{aligned}
Z_{\dot{z}}= & \int_{L} N(x) d x=\left[5.4 \frac{C_{w}}{C_{p}} \sqrt{\frac{B}{2 d}}-4.7\right] \frac{\Delta}{\sqrt{g L}} \\
Z_{z} & =\rho g A_{w} \\
Z_{\ddot{\theta}} & =-\lambda_{33} x_{G} \cong 0 \\
Z_{\dot{\theta}}= & -\int_{L} N(x) x d x+\left(\lambda_{33}-\lambda_{11}\right) u \\
& =\frac{\rho g \nabla \cdot G M_{L}}{u}+\left(\lambda_{33}-\lambda_{11}\right) u \\
Z_{\theta}= & u \int_{L} N(x) d x=u Z_{\dot{z}}
\end{aligned}\right.
$$

Pitch hydrodynamic derivative:

$$
\left\{\begin{aligned}
M_{\dot{\theta}} & =\int_{L} N(x) x^{2} d x=\frac{0.08 \Delta L^{2}}{\sqrt{g L}} \cdot \frac{B}{2 d} \\
M_{\theta} & =\rho g \nabla G M_{L} \\
M_{\ddot{z}} & =-\lambda_{11} x_{G} \cong 0 \\
M_{\dot{z}} & =-\int_{L} N(x) x d x-\left(\lambda_{33}-\lambda_{11}\right) u \\
& =\frac{\rho g \nabla G M_{L}}{u}-\left(\lambda_{33}-\lambda_{11}\right) u \\
M_{z} & =\rho g A_{w} x_{f}
\end{aligned}\right.
$$

where:

$C_{w}$ is waterplane coefficient;

$C_{p}$ is prismatic coefficient;

$\rho$ is density of sea water;

$g$ is gravitational acceleration;

$G M_{L}$ is longitudinal metacentric height;

$A_{w}$ is waterplane area;

$x_{f}$ is $\mathrm{x}$ value of center of floatation.

\subsection{Wind Force Module}

When a ship is in motion in the sea, effect of wind on the area of ship which is above waterline, leads to heading deviation and difficulties for manipulation. When sailing in the harbor at low speed, the impact of wind on the ship's steering is particularly obvious, making maneuvering more complicated. So, calculation for wind force plays an important role in accuracy of simulation.

Wind speed above ocean surface is termed as $U_{T}$ and wind angle is $\Psi_{T}$. Wind speed $U_{T}$ and wind angle $\Psi_{T}$ defined in earth-fixed reference fame are also called true wind speed and true wind angle. Definition of true wind angle is: northern wind is 0 degree, eastern wind is 90 degree, and arrangement of $\Psi_{T}$ is from 0 to 360 . Wind speed and wind angle observed in body-fixed reference fame are relative wind speed and relative wind angle. Relative wind speed is termed as $U_{R}$, with the value of wind from portside being positive.

Relationship of turn wind speed $U_{T}$, ship speed $V$ and relative wind speed $U_{R}$ is:

$\vec{U}_{R}=\vec{U}_{T}-\vec{V}$

Rotating the body-fixed frame we get

$u_{R}=-u-U_{T} \cos \left(\Psi_{T}-\varphi\right)$

$v_{R}=-v-U_{T} \sin \left(\Psi_{T}-\varphi\right)$

where: $u_{R}, v_{R}$ are the values of $U_{R}$ on $x, y$ axis in bodyfixed frame, $U_{R}^{2}=u_{R}^{2}+v_{R}^{2}$.

In the body-fixed frame, value of wind angle $\alpha_{R}$ from portside is positive, so:

$\begin{cases}\alpha_{R}=\arctan \left(-\frac{v_{R}}{u_{R}}\right)+\operatorname{sgn}\left(\pi, v_{R}\right) & u_{R}>0 \\ \alpha_{R}=\arctan \left(-\frac{v_{R}}{u_{R}}\right) & u_{R}<0\end{cases}$

Wind forces and moments acting on ships are calculated by (16):

$\left\{\begin{array}{l}X_{\text {WIND }}=0.5 \rho_{a} A_{f} U_{R}^{2} C_{w x}\left(\alpha_{R}\right) \\ Y_{W I N D}=0.5 \rho_{a} A_{s} U_{R}^{2} C_{w y}\left(\alpha_{R}\right) \\ N_{W I N D}=0.5 \rho_{a} A_{s} L_{O A} U_{R}^{2} C_{w n}\left(\alpha_{R}\right)\end{array}\right.$

where:

$\rho_{a}=1.204 \mathrm{~kg} / \mathrm{m}^{3}$ is the air density

$L_{O A}$ is the overall length

$A_{f}, A_{s}$ are areas of longitudinal and lateral projection above water surface.

$C_{w x}\left(\alpha_{R}\right), C_{w y}\left(\alpha_{R}\right), C_{w n}\left(\alpha_{R}\right)$ are coefficients of longitudinal and lateral wind resistance and rolling moment, obtained by empirical equations or wind tunnel tests $[11,12]$.

\subsection{Current Force Module}

Similar to wind, sea current forces and moments are calculated by (17): 


$$
\left\{\begin{array}{l}
X_{\text {current }}=0.5 \rho U_{c}^{2} A_{c}^{f} C_{c x}(\beta) \\
Y_{\text {current }}=0.5 \rho U_{c}^{2} A_{c}^{s} C_{c y}(\beta) \\
N_{\text {current }}=0.5 \rho U_{c}^{2} A_{c}^{s} L_{O A} C_{c n}(\beta)
\end{array}\right.
$$

where:

$\rho_{a}=1.025 \times 10^{3} \mathrm{~kg} / \mathrm{m}^{3}$ is water density

$L_{O A}$ is the overall length

$A_{c}^{f}, A_{c}^{s}$ are areas of longitudinal and lateral projection below water surface.

$C_{c x}(\beta), C_{c y}(\beta), C_{c n}(\beta)$ are coefficients of longitudinal and lateral current resistance and rolling moment, obtained by wind tunnel tests.

\subsection{Wave Force Module}

In order to meet real-time simulation, for obtaining value of wave force, hydrodynamic software is applied to calculate wave force transfer function in different ship speed, heading angle and wave frequency [13]. In real-time simulation, value of wave force is calculated in each step by interpolation algorithm:

$F_{\text {wave }}=H(\omega, \theta) \zeta(\omega, \theta, x, y, t)$

where:

$H(\omega, \theta)$ is wave force transfer function as a function of wave frequency $\omega$ ship heading angle $\theta$;

$\zeta(\omega, \theta, x, y)$ is wave height.

Since real sea wave is extremly irregular and wave height, wave length and period are randomly varying, equation for regular wave is invalid. Assuming that irregular wave $\zeta(\omega, \theta, x, y)$ is composed of large number of unit waves with various wavelengths, wave amplitudes and random phases, irregular wave is expressed as $[14,15]$ :

$$
\begin{aligned}
\zeta(\omega, \theta, x, y, t)= & \sum_{i=1}^{n} \sum_{j=1}^{m} A_{i, j} \cos \left(k_{i} x \cos \theta_{j}\right. \\
& \left.+k_{i} y \sin \theta_{j}-\omega_{i} t+\varphi\right)
\end{aligned}
$$

For utilizing spectrum analysis to forecast the performance of ship in irregular waves, firstly, wave/wind spectrum density is estimated of voyage areas. Researchers have proposed a variety of ocean wave spectrum expressions based on a number of observational and theoretical works, for example, Bretschneitder spectrum, Pierson-Moskowitz spectrum, ITTC parameter spectrum, JONSWAP spectrum and Torsethaugen spectrum. In this paper, a doubleparameter spectrum method which is recommended by ITTC and ISSC is applied. This spectrum is applicable to fully developed waves, developing waves and waves with swell:

$S(\omega)=\frac{173 H_{1 / 3}^{2}}{T_{1}^{4} \omega^{5}} \exp \left(-\frac{691}{T_{1}^{4} \omega^{4}}\right)$

where:
$H_{1 / 3}$ is one-third significant wave height;

$T_{1}$ is the spectral centroid period, close to average period from observation where $T_{1}=2 \pi m_{0} / m_{1}$; it can also be termed as peak spectral period, in relation with peak spectral period: $T_{0}=1.2965 T_{1}$;

$\omega$ is angular frequency.

Lee carried out statistics for sea state of Atlantic and North Pacific in 1985, offering values of significant height, spectral peak period and wind speed and possibility for each sea state. More details are listed in reference [16].

Since ITTC double-parameter spectrum is $1-D$, but real sea wave is 3-D, energy is distributed within a broad range of frequencies and directions, in order to characterize the wave as being direction-dependent. ITTC recommends a direction spectral function:

$$
D(\theta)=\frac{2}{\pi} \cos ^{2}\left(\frac{\theta}{2}\right) \quad|\theta| \leq \frac{\pi}{2}
$$

If frequency distribution and direction distribution of wave energy are independent and linear, distribution of wave energy can be termed by product of sea wave spectral function and direction spectral function:

$$
\begin{aligned}
A_{i, j}=S(\omega) D(\theta)= & \frac{173 H_{1 / 3}^{2}}{T_{1}^{4} \omega^{5}} \exp \left(-\frac{691}{T_{1}^{4} \omega^{4}}\right) \\
& \cdot \frac{2}{\pi} \cos ^{2}\left(\frac{\theta}{2}\right)
\end{aligned}
$$

\subsection{Thruster Module}

A model is applied to calculate the thruster of propeller $T$ and torque $Q$ in real conditions:

$\left\{\begin{array}{l}T=\left(1-t_{p}\right) \rho n^{2} D^{4} K_{T} \\ Q=\left(1-t_{p}\right) \rho n^{2} D^{5} K_{Q} \\ J_{p}=\left(1-\omega_{p}\right) U /(n D)\end{array}\right.$

where:

$J$ is the advance coefficient;

$w_{p}$ is the wake current coefficient;

$t_{p}$ is the thrust deduction coefficient;

$n$ is the propeller speed;

$D$ is the propeller diameter;

$K_{T}, K_{Q}$ is the thrust coefficient and torque coefficient, obtained by propeller test in open water;

\section{RAPID DEVELOPMENT FOR MOTON SIMU- LATION SYSTEM}

For real-time communication, firstly mutual transmission is achieved by UDP protocol. A real-time 


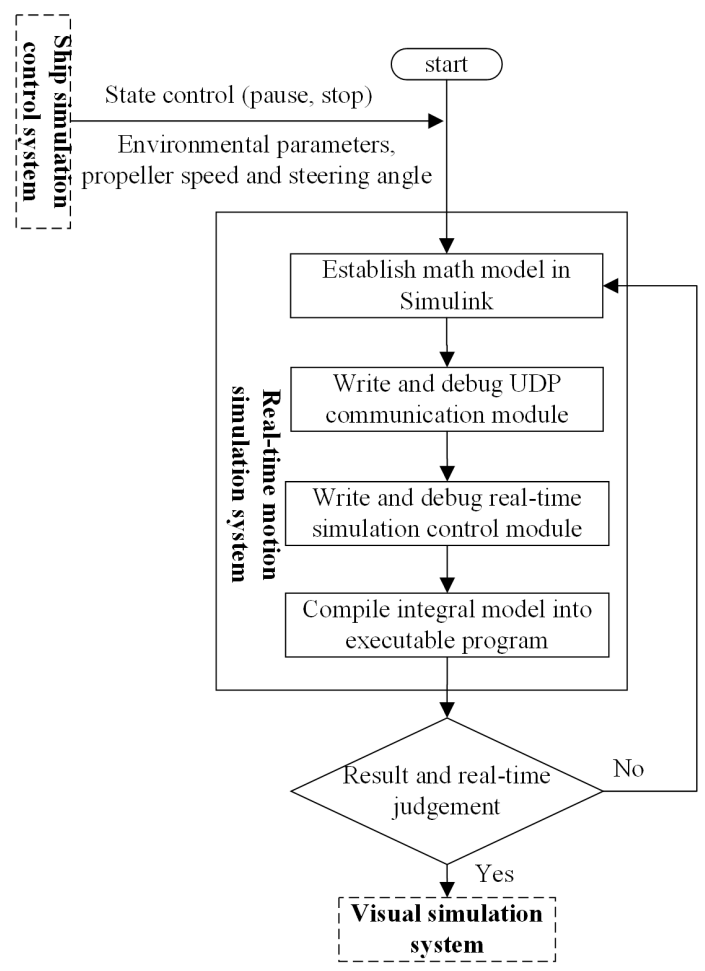

Fig. (3). The flow chart of real-time simulation system.
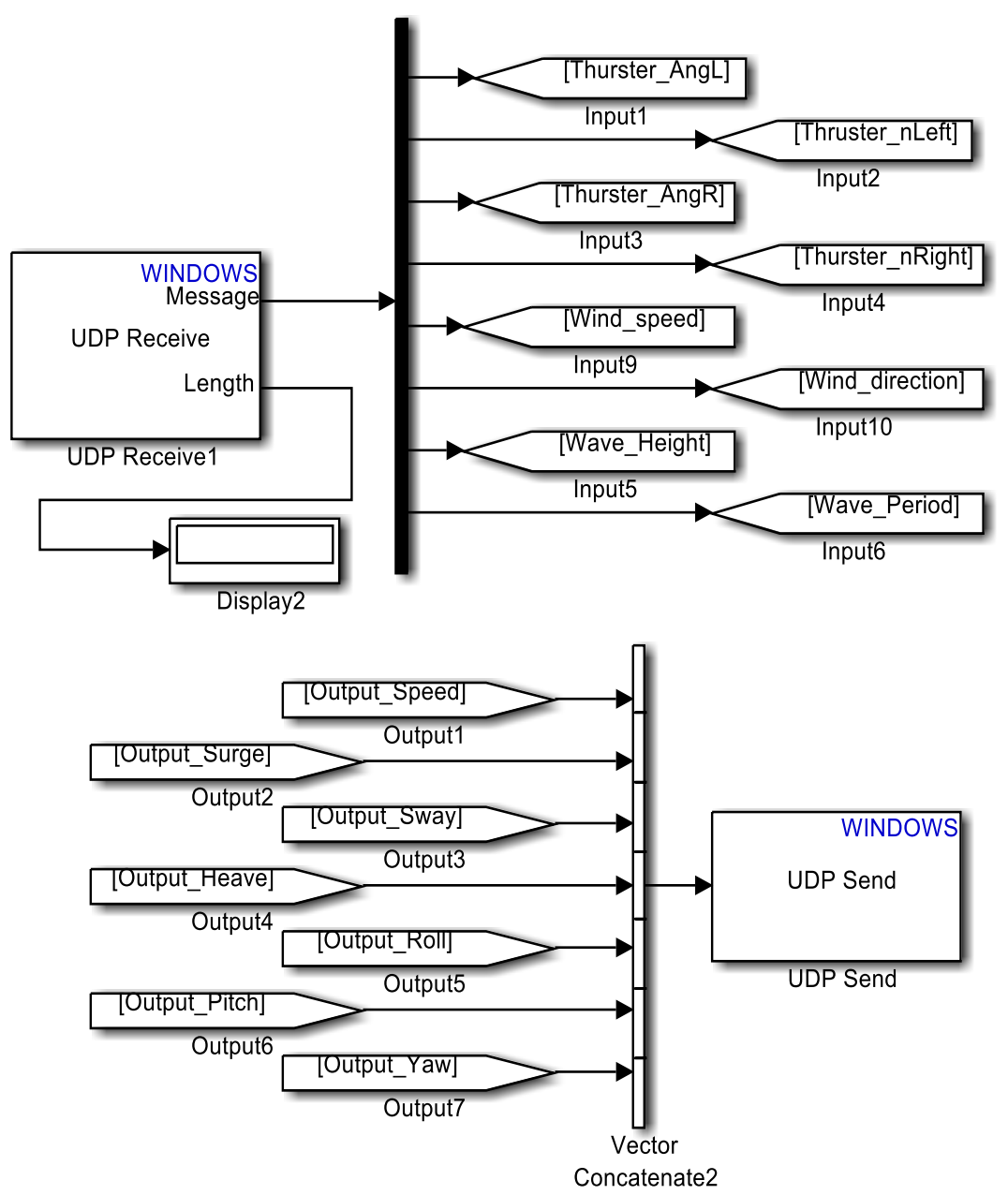

Fig. (4). UDP communication model development. 
communication module by S-Function is established and the module is added into Simulink model. As a result, controlling real-time calculation for simulation data is achieved. Fig. (3) shows the flow chart of real-time simulation system.

\section{UDP COMMUNICATION}

UDP communication module contains a data receiver module and a data transmission module. After receiving realtime control data from simulation control system, motion simulation system analyzes the data packet and then carries out ship motion calculation and finally, data transmission module transfers the data of ship motion to visual simulation system. Fig. (4) represents UDP communication module in Simulink.

\section{RESULTS AND DISCUSSION}

Inputting parameters into ship simulation control system, wave average period is $T=7.2 \mathrm{~s}$, wave significant height is $H_{1 / 3}=2.5 \mathrm{~m}$, wind speed $U_{\text {wind }}=18 \mathrm{kn}$, wave angle and wind angle both are $\psi=45^{\circ}$, speed of left/right propeller is $n=180 \mathrm{rpm}$. When simulation time is $800 \mathrm{~s}$, steering angle changes to $\theta=15^{\circ}$. Figs. (5-7) show the results of motion simulation.

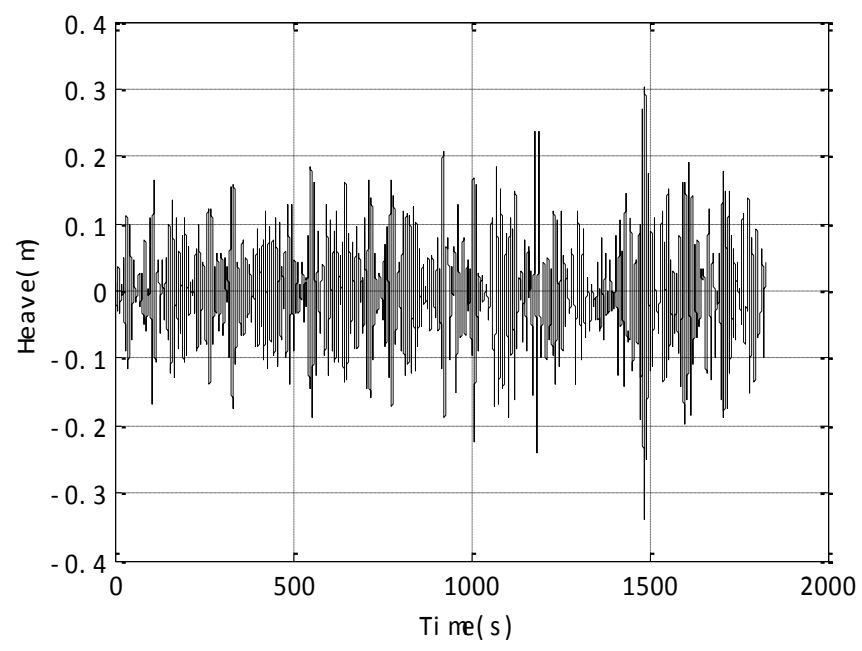

Fig. (5). Heave angle curve.

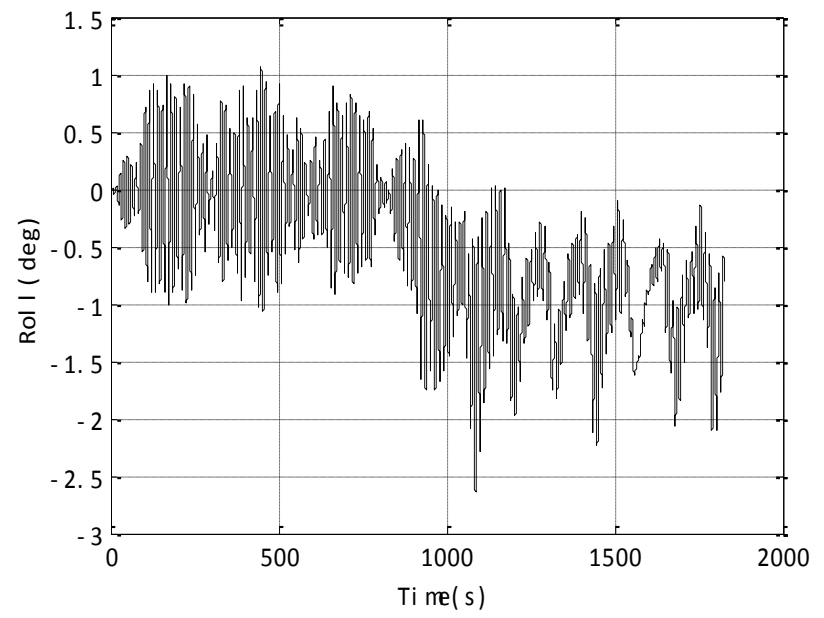

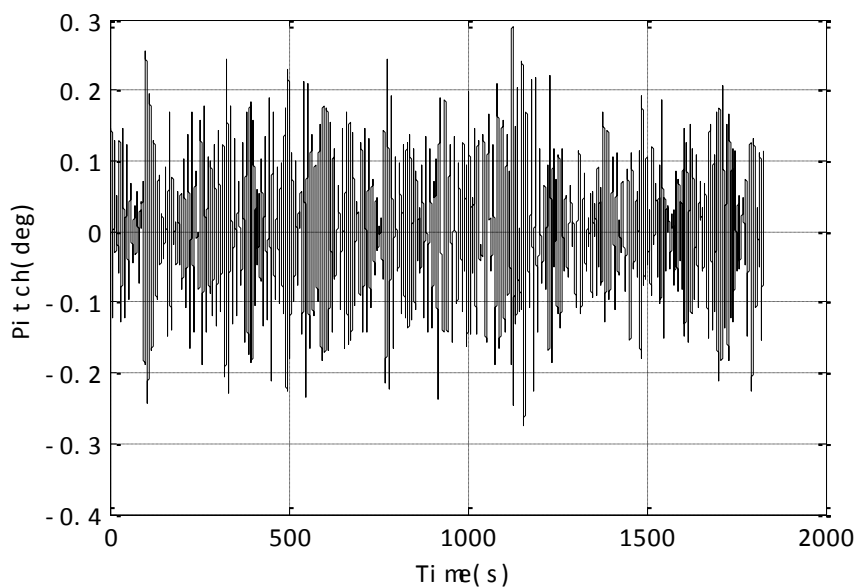

Fig. (7). Pitch angle curve.

A ship maintains direct sailing in waves in $[0 s, 800 s]$, at peak value of heave in $0.18 \mathrm{~m}$, while peak value of roll angle is 1 degree, and peak value of pitch angle is 0.25 degree. At 800 s, ship begins to change steering angle, peak value of heave increases to approximately $0.3 \mathrm{~m}$, peak value of rolling increases to 2.5 degree, with slight change occurring on the pitch. Fig. (8) is the final result of simulation shown in visual system:

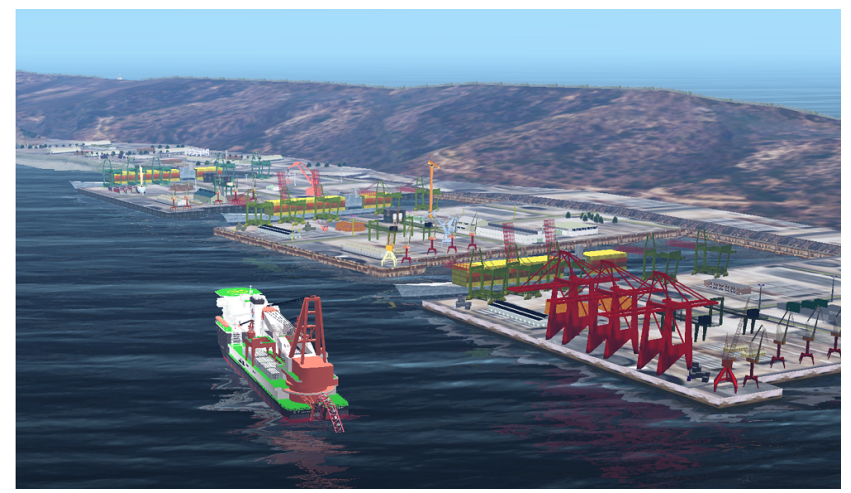

Fig. (8). Visual scene display.

\section{CONCLUSION}

In this paper, a project ship was taken as a sample, and a method based on Simulink was introduced for developing a real-time simulation system. After verifying real-time communication, visual-refresh rate of visual system reached $100 \mathrm{HZ}$, fully satisfying the requirement of visual real-time. This method avoids tedious work for manually changing codes, eliminates the process of compiling codes for communication and real-time simulation control. It also greatly facilitates the development and integration of the system, shortening the process of developing real-time simulation system.

\section{CONFLICT OF INTEREST}

The authors confirm that this article content has no conflict of interest.

Fig. (6). Roll angle curve. 


\section{ACKNOWLEDGEMENTS}

This work is supported by the National Science and Technology Major Project of the Ministry of Science and Technology of China (No. 2011ZX05027-002), China Scholarship Council and the Technology Project of China National Offshore Oil Corporation (No. CNO0C-KJ 125 ZDXM 05 GC 00 GC 2013-04).

\section{REFERENCES}

[1] Z. Tao, "Research on network communication between Simulink and $\mathrm{VC}++$ based on S-function," Modern Electronics Technique, vol. 36, no. 13, pp. 108-111, 2013.

[2] C. Salzmann, D. Gillet, and P. Huguenin, "Introduction to real-time control using LabVIEWTM with an application to distance learning," International Journal of Engineering Education, vol. 16, no. 5, pp. 372-384, 2000.

[3] B. Netten, and H. Wedemeijer, "Testing cooperative systems with the MARS simulator," In: Proc. Intelligent Transportation Systems (ITSC), $201013^{\text {th }}$ International IEEE Conference on, IEEE, pp. 186-191, 2010.

[4] A. M. Abdullah, and M. A. Dia, "Creating Real-Time operation System Based on xPC Target Kernel," International Journal of Recent Technology and Engineering, vol. 2, no. 4, pp. 143, 2013.

[5] SNAME, "The Society of Naval Architects and Marine Engineers," Nomenclature for Treating the Motion of a Submerged Body Through a Fluid. In: Technical and Research Bulletin No. 1-5., New York, USA 1950.
[6] T. I. Fossen, "Handbook of marine craft hydrodynamics and motion control," Wiley, 2011.

[7] Z. Zhou, Z. Sheng and W. Fen, "Multipurpose cargo handling forecast calculation," Ship Engineering, no. 6, pp. 21-29+36+24, 1983.

[8] J. Li, "Ship seakeeping", Harbin Shipbuilding Engineering Institute Press, Harbin, 1992.

[9] X. Jia, and Y. Yang, "Ship motion mathematical model", Dalian Maritime University Press: Dalian, 1999.

[10] F. Tasai, "On the Damping Force and Added Mass of Ships Heaveing and Pitching. ", Shipbuilders' Association Proceedings, vol. 105, pp. 47-56, 1959.

[11] T. I. Fossen, "How to Incorporate Wind, Waves and Ocean Currents in the Marine Craft Equations of Motion," IFAC $M C M C^{\prime} 12,2012$.

[12] M. R. Haddara, and S. C. Guedes, "Wind loads on marine structures," Marine Structures, vol. 12, no. 3, pp. 199-209, 1999.

[13] Z. Chuang, and S. Steen, "Speed loss due to seakeeping and maneuvering in zigzag motion," Ocean Engineering, vol. 48, pp. $38-46,2012$.

[14] Z.B. Sheng, and Y. Z. Liu, "Principle of ship," Shanghai Jiao Tong University Press: Shanghai, 2004

[15] Y. F. Zan, H. D. Zhi, L. Song, and W. Sun, "The Numerical Simulation Research of Three Dimensional Irregular Wave Based on Wave Spectrum", Applied Mechanics and Materials, vol. 397, pp. 643-647, 2013.

[16] W. T. Lee, S. L. Bales, and S. Sowby, "Standardized wind and wave environments for North Pacific Ocean Areas," DTIC Document, No. DTNSRDC/SPD-0919-02, 1985. 